\title{
Conceptualisation linguistique : du manuscrit au texte. Contribution à l'étude des spécificités de l'écriture scientifique
}

\author{
Irène Fenoglio \\ Institut des Textes et Manuscrits Modernes, CNRS/ENS, Paris \\ Irene.Fenoglio@ens.fr
}

L'écriture linguistique est actuellement l'objet de divers travaux (analyses de discours, études de genres, travaux de terminologie, genèse de textes publiés) mais rares, encore, sont ceux qui, pour comprendre l'écriture scientifique et la conceptualisation de notions, s'attachent à l'aspect génétique et processuel de l'écriture en train de se faire, visible sur les manuscrits. L'objectif de cette recherche est de contribuer à ouvrir ce champ de travail de l'écriture en production, d'une grande complexité où les "brouillons" permettent d'observer la façon dont un auteur scientifique (en l'occurrence linguiste) s'y prend pour formuler son discours théorique, pour textualiser une notion linguistique. Ce type d'étude devrait compléter utilement les travaux accomplis sur les textes produits, finis, publiés.

La présente contribution propose des éléments de modélisation du discours scientifique linguistique à partir d'un corpus de manuscrits, ceux correspondant à l'écriture de l'article « Le langage et l'expérience humaine » d'Emile Benveniste.

\section{Choisir un corpus de travail : l'avant-texte de l'article « Le langage et l'expérience humaine " d'Émile Benveniste}

Choisir un corpus de travail, d'une façon générale, n'est pas chose aisée, il s'agit d'une étape où l'enjeu scientifique, autrement dit la question posée au départ, doit faire bonne mesure avec tout un ensemble de composantes et de limites qui ne dépendent pas ou peu du chercheur: matériau disponible, méthodes d'investigation, travaux antérieurs, etc.

La question précise à laquelle je tente de répondre est la suivante : est-ce qu'observer la façon dont un scientifique s'y prend pour formuler sa théorie - et donc les concepts qui la sous-tendent - permet de comprendre le processus d'écriture scientifique, en l'occurrence, linguistique ? Autrement dit est-ce que le recueil des traces de textualisation du discours scientifique et leur analyse - elle-même linguistique peut permettre de comprendre la façon dont un discours linguistique se construit et d'en exhumer les traits distinctifs et constitutifs?

Il est clair que si nous pensons que cette expérience doit être menée, il faut trouver le corpus adéquat, c'est à dire un "manuscrit" au sens large du terme, un avant-texte, au sens généticien du terme, quelle qu'en soit la "forme“. Qu'est-ce qu'un avant-texte ? Jean Bellemin-Noël est le premier à avoir défini cette notion, en deux temps. Dans un premier temps, il le définit comme «l'ensemble constitué par les brouillons, les manuscrits, les épreuves, les "variantes", vu sous l'angle de ce qui précède matériellement un ouvrage quand celui-ci est traité comme un texte, et qui peut faire système avec lui ${ }^{1}$. Dans un deuxième temps, il inclura dans la compréhension de cette notion le travail critique du chercheur qui d'un ensemble empirique de documents fait un "dossier" d'éléments significatifs et ordonnés. L'avant-texte devient «quelque chose de construit [...] qui n'existe nulle part en dehors du discours critique qui le produit $»^{2}$. L'ensemble informel de documents dont la spécificité est essentiellement temporelle prend alors un statut scientifique: l'hypothèse d'un ordre génétique est proposée et va soutenir une interprétation du processus d'écriture du texte. Qu'est-ce qu'un "ordre génétique" ? Il s'agit de l'ordonnancement chronologique des traces des différentes états d'écriture depuis le "premier jet" jusqu'au texte final.

Vu l'état naissant de ce type de travaux que je pourrais qualifier de "linguistique génétique" ou de "génétique énonciative" portant sur des manuscrits linguistiques - corpus inhabituels et tout à fait 
nouveaux en génétique du texte - je n'ai choisi qu'un seul auteur, à charge pour moi et d'autres chercheurs intéressés de continuer l'étude sur la base comparative d'un corpus multi auteur. Le fonds Benveniste de la BnF qui, aujourd'hui, est à peu près le seul, après celui de Saussure, à être exploité par quelques linguistes est d'une grande pertinence en regard de la question posée.

En effet, nous y sommes confrontés à trois genres au moins d'écriture scientifique : l'écriture d'article, l'écriture de communication scientifique à des Congrès, l'écriture propre à la préparation de cours (en l'occurrence cours à l'EPHE et au Collège de France), sans compter les carnets de travail et d'observation pour la description de langues, des plus rares aux plus connues.

L'écriture d'article m'est apparu comme la plus pertinente, la plus adaptée à l'enjeu de cette contribution, car, d'une part, il s'agit forcément d'écriture et non pas de notations ou d'aide-mémoire destinées à soutenir un discours oral comme pour un cours, voire une communication, et, d'autre part, le processus de textualisation y est conduit jusqu'à son terme ; l'observation génétique d'un fait textuel particulier comme la conceptualisation y trouve alors un espace de travail approprié.

\subsection{Habitus de travail du Linguiste}

Quelques travaux précédents rendant compte d'incursions dans le fonds Benveniste (Fenoglio, 2009a, 2009 b, 2010) permettent de comprendre la façon dont le linguiste fonctionne habituellement pour l'écriture d'un article, car, en effet, nous parvenons à peu près, chaque fois, à réunir, disséminés dans différentes boites ou chemises d'archives, les mêmes éléments : des notes manuscrites ${ }^{3}$, puis un brouillon manuscrit parfois suivi d'une mise au net manuscrite avec instructions de mises en pages, souvent une dactylographie avec un double carbone, quelquefois un ou plusieurs jeux d'épreuves.

La réitération d'une même suite d'éléments manuscrits et de documents pour de nombreux articles permet d'imaginer la façon dont Benveniste écrivait. Benveniste ne se passait jamais de "notes", nombreuses, sur divers supports, parfois répétitives ou, comme j'ai pu le montrer (2009a), ruminatives. Leur observation est essentielle à la compréhension de l'élaboration scripturale et permet l'analyse du passage des notes au brouillon qui reste un moment essentiel pour la textualisation théorique.

Il n'est pas rare, sur l'ensemble des dossiers manuscrits de Benveniste, de voir le contenu d'une note repris sur plusieurs folios : un engagement ruminatif dans l'écriture. En ce sens, Benveniste expose luimême dans son faire, et sans le savoir, l'idée qu'il défend selon laquelle la pensée n'existe pas préalablement au discours qui l'exprime : une écriture hésitative, répétitive, pensante où la répétition est la marque à la fois de l'hésitation et de l'insistance. Hésitation pour continuer de rechercher la formulation la plus adéquate. Insistance car la pensée est là qui fraye dans le fil des mots son chemin.

La démarche est cumulative et progressive à la fois. La « rumination » permet de garder tous les éléments essentiels, de les reprendre, de les faire circuler sur le papier, mais progressivement, l'ensemble avance vers un fil discursif décanté et ordonné. Les notes préparatoires réfléchissent les éléments qui constitueront l'enjeu du discours théorique de l'article : son cadre et sa problématique.

L'observation - encore trop rapide et partielle - de manuscrits d'Antoine Meillet ${ }^{4}$ nous permet déjà de constater des analogies dans les habitus de travail des deux linguistes : écriture de notes de format et style divers avant l'écriture en continu d'un brouillon d'article. Il est clair que si cette façon de faire se rencontrait chez d'autres linguistes, nous pourrions en tirer un trait commun caractérisant le processus d'écriture en linguistique théorique. Cela reste donc à suivre, avec des observations plus larges en sciences humaines. 


\subsection{Composition du manuscrit correspondant à l'article «Le langage et l'expérience humaine »}

Voici la composition du dossier relatif à l'article que nous étudions ici, tel que j'ai pu la reconstituer ; les différents éléments qui le composent sont distribués dans deux enveloppes différentes, provenant de deux cartons différents (enveloppe 139 de la boite 46 et enveloppe 179 de la boite 50) :

$1-$ Notes $^{5}$ : il y en a peu dans ce dossier comparativement à d'autres ${ }^{6}$.

2 - Un premier brouillon très "brouillonné"7 [désormais B1]

Les premiers feuillets sont numérotés par Benveniste de 1 à 33 et signés « $\mathrm{E} B$ » à la dernière page (p. 33, $f^{\circ} 535$ ).

3 - Un brouillon mis au net ${ }^{8}$ [désormais B2]

Ce brouillon est numéroté par Benveniste de la page 1 à 33

Le premier feuillet jaune, non numéroté mais correspondant à la page 1, a très certainement été écrit en fin de rédaction car son support est constitué du même papier jaune que celui qui porte la fin de l'article

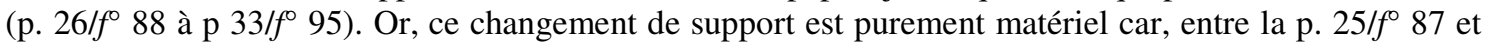
la p $26 / f^{\circ} 88$, une très longue phrase se continue tout d'une haleine, une seule pause intervenant au moment de la rature de la fin :

«si je raconte ce qui "m'est arrivé", le passé auquel je [changement de page] me
réfère n'est défini que par rapport au présent de mon acte de parole, mais l'acte de
parole surgit de moi et que personne autre [sic] ne peut parler par ma bouche non plus
que voir par m6es yeux ou éprouver ce que je sens, c'est à moi seul que ce "temps" se
rapportera et c'est ma seule expérience qu'il erdonnera organisera. »

4 - Double carbone du tapuscrit correspondant à l'article publié

On peut noter que le nombre de pages de B1 et de B2 (numérotées par Benveniste) est exactement identique : 33. Pourtant, nous le verrons, sept pages de $\mathrm{B} 1$ sont réduites à un seul paragraphe au début de B2. A partir de la p. $13\left(f^{\circ} 515\right)$ pour B1, p. $5\left(f^{\circ} 67\right)$ pour B2, et jusqu'à la fin, il n'y a, en gros, que des variantes de style, c'est-à-dire que B2 apparait comme une véritable mise au net de B1 avec une écriture plus aérée, plus large; d'où le même nombre de pages malgré la suppression de 7 pages (sur laquelle nous reviendrons).

Nous n'utiliserons pas, ici, la dactylographie car elle ne porte aucune correction manuscrite, cela n'aurait pas d'intérêt génétique, le texte qu'elle porte est identique au deuxième brouillon dont elle enregistre le dernier état.

Dans ce dossier, les notes préparatoires à l'écriture retrouvées ne sont pas nombreuses; on peut en compter six : folios 497, 499, 500, 501, 502, 503. A les examiner, nous voyons que dans chacune est pointée, de façon programmatique, une difficulté théorique. Nous n'avons aucun moyen de savoir dans quel ordre les notes ont été iécrites; les numéros figurant sur les fiches n'étant pas de la main de Benveniste, nous pouvons juste noter que les folios 502 et 503 se présentent sur des supports semblables : petites fiches jaunes rectangulaires (semblables à nos actuels post-it). J'ai considéré que la fiche 503 devait avoir été écrite avant la 502. Nous pouvons noter aussi que la note du $f^{\circ} 500$ pourrait être la suite de $f^{\circ} 499$.

Nous allons donc parcourir ces documents de genèse en essayant de pointer, chaque fois, l'opération de conceptualisation à laquelle ils correspondent. En attendant de les reprendre de façon plus systématique en 3., nous les indiquerons entre crochets et en italique. 


\section{Un exemple de conceptualisation scientifique : comment le mot "expérience" devient une notion théorique}

\subsection{L'incipit de l'article: genèse de l'émergence d'une notion et du positionnement épistémique d'un auteur}

\subsubsection{Les notes}

Voyons la note du $f^{\circ} 501$

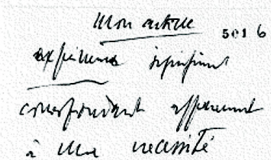

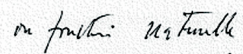

$$
\begin{aligned}
& \text { et insiefundued } \\
& \text { tonce rekminats } \\
& \text { conltula Sipini } \\
& \text { fer l'aptentementer } \\
& \text { la vialite jar la } \\
& \begin{array}{l}
\text { sups, sni insertion } \\
\text { fam le reel }
\end{array}
\end{aligned}
$$

Mon article

expérience signifiant correspondant apparemment à une nécessité ou fonction naturelle et indépendante de toute détermination culturelle. Défini par l'affrontement de la réalité par le sujet, son insertion dans le réel. ${ }^{9}$

L'indication soulignée «Mon article » montre bien qu'il s'agit d'une note préparatoire où le linguiste s'explicite pour lui-même la notion qu'il veut développer. Auto-réflexion à la fois épistémologique et méthodologique, ce n'est qu'en écrivant qu'il la pense. Nous pouvons noter que le terme «expérience » est pointé d'emblée, ce terme pose question : trop commun, trop banalisé, il y a nécessité pour le linguiste de présenter à lui-même la raison de son emploi, donc sa signification pour lui, théoricien du langage. La note est constituée de deux phrases participiales, sans verbes actifs : un participe présent «signifiant » un participe passé « défini »; ces deux participes, bien que marquant tout deux un accompli indiquent très précisément l'opération de conceptualisation en train de se faire: définir, préciser le sens du terme employé. L'auteur avance vers une spécification du terme, veut définir, mais il est encore loin d'y parvenir ; en effet, l'ensemble de cette note demeure peu clair.

[Pointer puis resserrer la détermination des termes employés pour la construction théorique]

La note du f ${ }^{\circ} 503$ pointe les deux éléments de l'expérience humaine qu'il développera dans l'article, de ce fait, je considère qu'elle a dû être écrite avant celle du f $f^{\circ} 502$ qui ne s'intéresse qu'au temps. Elle constitue à la fois un «support de réflexion» et un «support de mémoire » (Fenoglio 2009, 27).

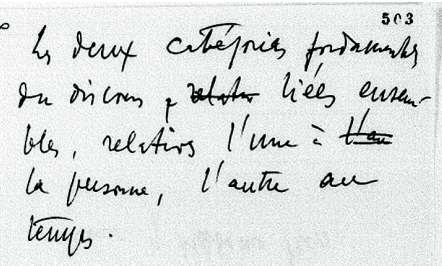

Les deux catégories fondamentales du discours, relati liées ensembles, relatives l'une à l'au la personne, l'autre au temps 

principe en vue de séparer, dans ce qu'on désigne par temps, ce qui relève <résulte> de la réflexion consciente et ce qui revient à la conceptualisation du langage

La note du $f^{\circ} 502$ correspond à un «support méthodologique » (Fenoglio 2009, 26). L'injonction est clairement marquée : l'infinitif «tracer» en est spécifique. On sait que le programme méthodologique annoncé aura été respecté dans l'écriture de l'article au point que le passage concernant l'expérience humaine du temps sera beaucoup plus important que celui concernant l'expérience intersubjective exprimée linguistiquement par les pronoms. On peut noter, par ailleurs, l'hésitation entre « relève » et « résulte ». Contrairement au passage « entend <désigne> », Benveniste ne tranche pas encore et garde les deux possibilités qui sont pourtant loin d'être identiques et d'offrir un paradigme lexical tel qu'on en trouve généralement sur les brouillons. Dans le premier cas, « relève », il note une source d'expression, dans l'autre cas, « résulte », il spécifie un lien de conséquence.

[Enoncer un programme d'opérations méthodologiques]

Nous allons pouvoir constater que pas moins de six étapes sont nécessaires à l'écriture du début de l'article.

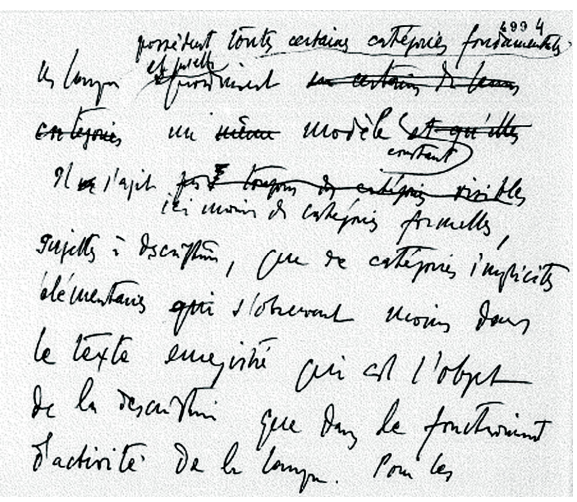

499

les langues <possèdent toutes certaines catégories fondamentales et qu'elles $>$ reproduisent encertines de leurs catégories un même modèle <constant> et qu'elles

Il ne s'agit <pas t toujours des catégories visibles> ici moins des catégories formelles, sujettes à description, que de catégories implicites élémentaires qui s'observent moins dans le texte enregistré qui est l'objet de la description que dans le fonctionnement d'activité de la langue. Pour les

Dans cette note où l'on voit la différenciation entre « texte enregistré » et «fonctionnement d'activité de la langue », Benveniste fait une distinction précise qui annonce la partition fondamentale chez lui entre langue et discours. «Texte enregistré » renvoie à discours et nous verrons plus loin que d'autres expressions de ce type sont essayées. Nous pouvons remarquer qu'après l'avancée d'une proposition que le linguiste suppose acceptable par tous: «les langues possèdent toutes certaines catégories fondamentales... » - elle est acceptable puisqu'elle marque l'héritage de Saussure - arrivent les spécificateurs «il s'agit de » et «ici »: Benveniste marque sa place, son apport, sa continuité à partir de l'héritage saussurien considéré comme commun à tous les linguistes.

[Marquer sa place particulière d'auteur à partir d'un héritage commun]

Les deux notes suivantes se tiennent sur une même feuille pliée en deux mais n'ont vraisemblablement pas été écrite dans la foulée. 

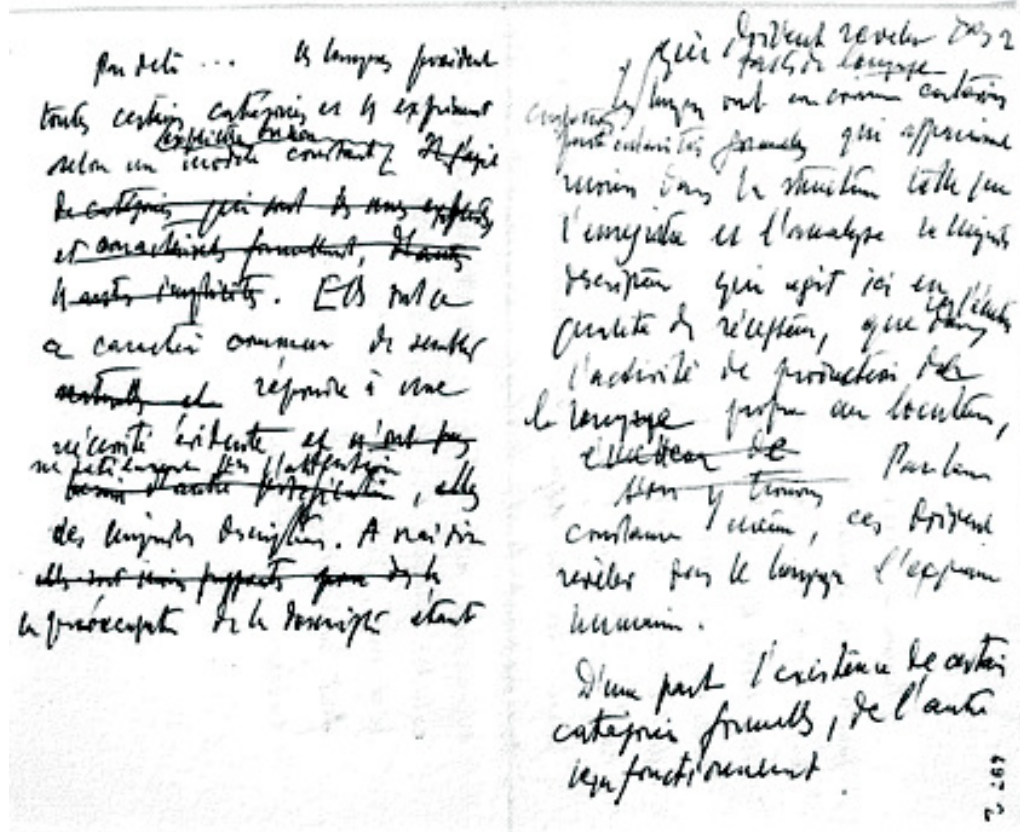

Par delà... les langues possèdent toutes certaines catégories et les expriment selon un modèle constant <explicite ou non>. H s'agit de catégories qui sont les unes explicites et caractérisées formellement, d'autres=les autres implicites. Elles ont ce ce caractère commun de sembler naturelles et répondre à une nécessité évidente et n'ont pass ne retiennent pas l'attention besoin d'antre justifican, elles-des linguistes descripteurs. A vrai dire elles sont moins frappantes pour des ta-la préoccupation de la description étant

Les langues ont en commun certaines particularités formelles <catégories certaines particularités formules $<$ catégories <qui doivent révéler des faits de langage >> qui apparaissent moins dans la structure telle que l'enregistre et l'analyse le linguiste descripteur qui agit ici en qualité de récepteur, que <chez l'émetteur> dans l'activité de production du/e <la> langage/ue propre au locuteur, <émetteur de>

Nous y trouvons Par leur constance même, ces doivent révéler dans le langage l'expérience humaine.

D'une part l'existence de certaines catégories formelles, de l'autre leur fonctionnement.

Par delà la réécriture insistante et ruminative des éléments déjà présents sur le $f^{\circ} 499$ vu précédemment, plusieurs remarques peuvent être faites :

- le passage de «description » à « linguiste descripteur ». Cette spécification est intéressante car on passe d'une activité commune à une fonction spécifique déterminant une catégorie de linguistes, cela signifierait : il y a des linguistes exclusivement descripteurs et il en existe d'autres parmi lesquels je me range, ici.

- l'hésitation entre langage et langue : «dt/e <la> langege/ue propre au locuteur» qui témoigne à la fois de l'instabilité conceptuelle de termes proches et de la volonté de précision du linguiste

- la dernière phrase laissée en suspend sur la note de droite marque la spécificité de l'auteur linguiste dont on sait qu'il va décrire, et ces catégories formelles, et leur fonctionnement dans «L'appareil formel de l'énonciation » : expression dont nous verrons plus loin qu'elle voit le jour dans le brouillon de cet article.

\subsubsection{Les brouillons : deux versions en $B 1, f^{\circ} 496$ et $f^{\circ} 498$ et une mise au net en $B 2 f^{\circ}$ 62}

Le folio 498, rangé initialement parmi les notes (Fenoglio, 2009, p. 33-35) me semble, par la présence du titre, entrer déjà dans le processus d'écriture de l'article. Par ailleurs, entre les deux versions de B1, j'ai 
estimé que celle du $f^{\circ} 496$ est antérieure à celle du $f^{\circ} 498$ par le fait que le terme « humaine » du titre est ajouté (encre bleue, sur écriture en noir). Le $f^{\circ} 498$ est plus proche de B2. Qu'on en juge :

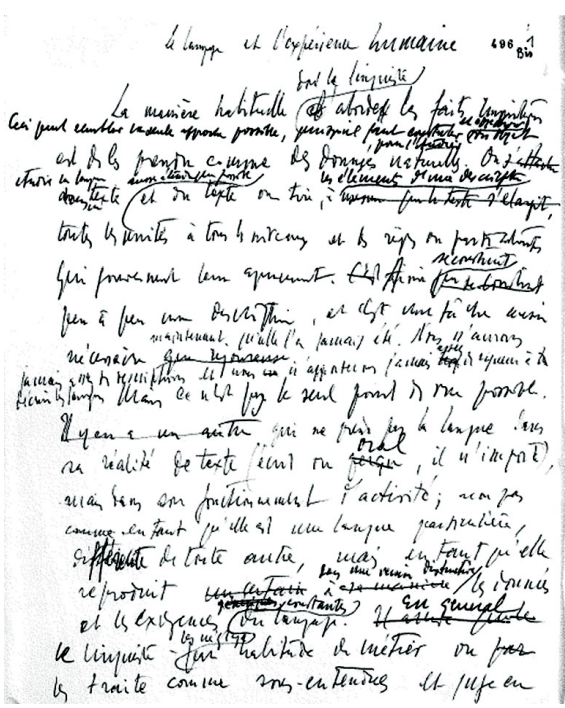

Le linguiste réapparaît sous une forme générique, il établit certes des descriptions, cependant nous ne sommes plus face au seul « linguiste descripteur » ce qui lui permet d'avancer que la description n'est pas « le seul point de vue possible », autrement dit que la description n'est pas la seule tâche du linguiste, il ouvre ainsi l'autre voie qu'il empruntera : catégoriser linguistiquement «l'expérience humaine ». [Différencier les tâches, les méthodes, les points de vue sur un même objet]

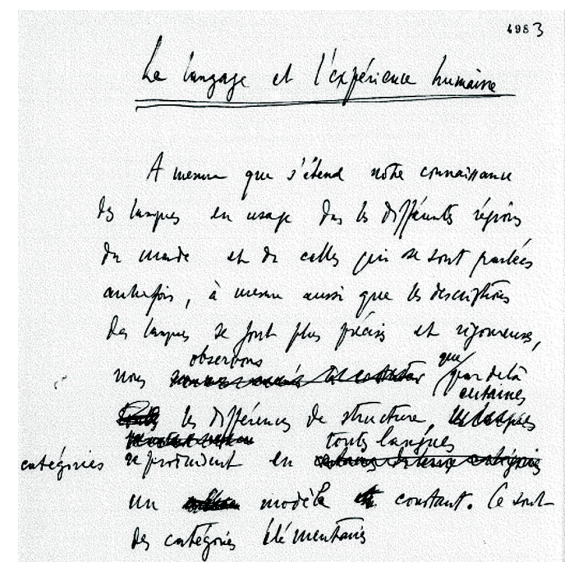
La manière habituelle <dont le linguiste $>$ d'aborder les faits linguistiques $<$ Ceci peut sembler une seule approche possible, puisqu'il faut constater <et observer > un objet pour l'étudier $>$ est de les prendre comme des données naturelles. On s'attache étudie <la langue >au/dans un texte aussi étendu que possible et du texte on tire বles éléments d'une description et du texte on tire, à mesure que le texte s'elargit $\checkmark$ les éléments d'une description>toutes les unités à tous les niveaux et les règles ou particularités qui gouvernent leur agencement. E'est a/Ainsi que se construit <se construit>-peu à peu une description, et c'est une tâche aussi nécessaire tue rigoureuse maintenant qu'elle l'a jamais été. <Nous n'aurons jamais assez de descriptions et nous ne n'apporterons jamais <assez> de rigueur à décrire les langues> Mais ce n'est pas le seul point de vue possible. Il y en an antre qui ne prend pas la langue dans sa réalité de texte (écrit ou perçu $<$ oral>, il n'importe), mais dans son fonctionnement d'activité; non pas comme en tant qu'elle est une langue particulière, distelférente de toute autre, mais en tant qu'elle reproduit un certain à sa manière < dans une version distinctive $>$ les données et les exigences du langage < génériques constantes> $t$ larive que te <En général> le linguiste les néglige par habitude de métier ou par les traite comme sous-entendues et juge en

Il est visible (même sans la couleur qui ferait apparaître de nombreux changements de stylo, autrement dit de reprises) que le folio précédent (496) est beaucoup plus «brouillon » que le $f^{\circ} 498$. Benveniste peine à introduire son propos, à énoncer son entrée en matière. Il semblerait qu'il ne s'y retrouve pas lui-même. Il reprend donc les fils et les remet sur le métier et l'on voit que le titre se stabilise au $f^{\circ} 498$, c'est-à-dire à la deuxième version du premier brouillon. Ici, le linguiste disparaît dans un «nous » dans lequel l'auteur 
s'englobe lui-même. «L'expérience humaine» se précise et se conceptualise linguistiquement en « certaines catégories », plus exactement en «modèle constant» dont toute langue trouvera les catégories. [Se situer comme auteur à l'intérieur d'une communauté]

Le premier feuillet de $\mathrm{B} 2\left(f^{\circ} 62\right)$ a vraisemblablement été écrit ou réécrit après que l'article ait été terminé car son support est une feuille épaisse, jaune, reconnaissable comme étant exactement semblable à celles qui supportent toute la fin de B2. Il est donc possible que le $f^{\circ} 498$ soit une première version de B2.

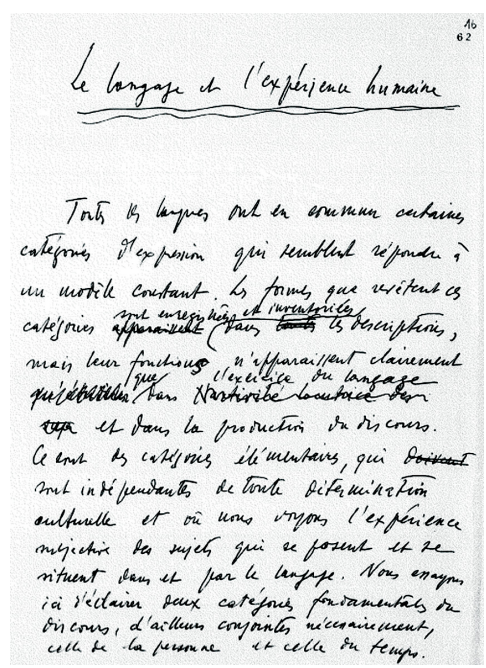

\section{Le langage et l'expérience humaine}

Toutes les langues ont en commun certainescatégories d'expression qui semblent répondre à un modèle constant. Les formes que revêtent ces catégories apparssent <sont enregistrées et inventoriées $>$ dans toutes les descriptions, mais leur fonctionn /s n'apparaissent clairement qu'éventuell <que> dans l'activité locutrice des suj $<$ l'exercice du langage $>$ et dans la production du discours. Ce sont des catégories élémentaires, qui doivent sont indépendantes de toute détermination culturelle et où nous voyons l'expérience subjective des sujets qui se posent et se situent dans et par le langage. Nous essayons ici d'éclairer deux catégories fondamentales $d u$ discours, d'ailleurs conjointes nécessairement, celle de la personne et celle du temps.

L'étape de la mise au net, B2 ( $\left.f^{\circ} 62\right)$, est marquée par l'entrée par une formule impersonnelle « toutes les langues », «les formes... », etc. Dans ce brouillon qui est très proche du texte final ${ }^{10}$, la pensée théorique s'affirme pour elle-même et d'abord. L'auteur linguiste qui se désigne par le «nous » en usage alors pour toute rédaction scientifique, n'intervient qu'après, à l'intérieur du cadre théorique posé pour indiquer son objectif et sa démarche : «nous essayons ici d'éclairer... » alors qu'à l'étape antérieure du $f^{\circ} 496$ (première version de B1) il apparaît d'emblée : «La manière dont le linguiste aborde... ». [Enoncer fermement sa tâche]

Nous pouvons, à cette dernière étape de l'écriture de l'incipit plusieurs éléments intéressants :

- il y a la fois distinction et équivalence entre les expressions « exercice du langage » et «production de discours »

- les «catégories élémentaires » de l'expérience humaine sont celles de «l'expérience subjective [...] dans le langage »

Nous sommes ainsi amenés à remarquer que deux axes de conceptualisation sont présents, ici, à partir de l'«expérience»: celle de «La subjectivité dans le langage» et celle de «L'appareil formel de l'énonciation ».

[Marquer son option théorique singulière à l'intérieur d'une communauté par l'indication d'une tâche particulière]

Nous venons de suivre la genèse de l'incipit où l'objet de l'article est cerné : catégoriser linguistiquement les éléments de «l'expérience humaine » pour conceptualiser ce terme d'expérience. Mais cela ne suffit pas à faire apparaître comment cette conceptualisation s'élabore. Rentrons plus avant dans l'avant-texte et suivons le terme « expérience. 


\subsection{La notion d' « expérience » : prémisses d'une théorie de l'énonciation}

\subsubsection{Mise en place difficile}

Dans $\mathrm{B} 1$, le $f^{\circ} 500$ doit être considéré comme une note préalable à la rédaction proprement dite de $\mathrm{B} 1$. Cette note est remarquable pour montrer la façon dont la mise en place de la notion d'expérience est difficile. Plusieurs expressions sont essayées : «présence de l'homme», «présence humaine », «vie affective », qui s'éloignent du cadre de catégories linguistiques. Le terme « expérience » n'arrive qu'en fin de page, dans une note non explicitée :

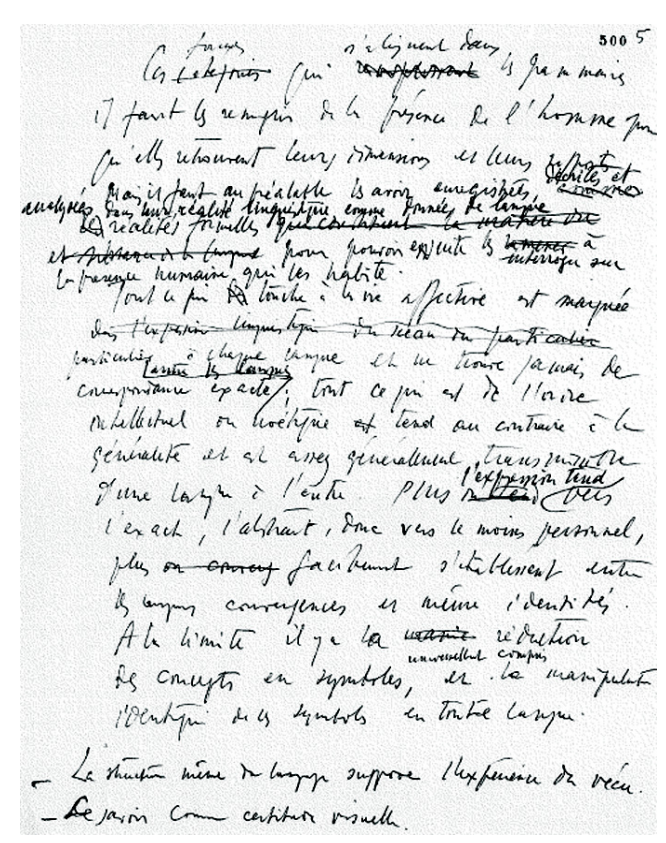

Ces éfógories $<$ formes $>$ qui remplissent $<$ 'alignent dans > les grammaires il faut les remplin de la présence de l'homme pour qu'elles retrouvent leurs dimensions et leurs rapports. Mais il faut au préalable les avoir enregistrées comme les réalités formelles qui constituent la matière ou la substance de ta langue <décrites et analysées dans leur réalité linguistique comme données de langue> pour pouvoir ensuite les ramer à <les interroger sur $>$ la présence humaine qui les habite.

Tout ce qui tes touche à la vie affective est marquée dans l'expression linguistique du sceau du particulier particulier à chaque langue et ne trouve jamais de correspondance exacte <entre les langues> ; tout ce qui est de l'ordre intellectuel ou noétique est tend au contraire à la généralité et est assez généralement transmissible d'une langue à l'autre. Plus on tend $<$ l'expression tend > vers l'exact, l'abstrait, donc vers le moins personnel, plus en converg facilement s'établissent entre les langues convergences ou même identités. A la limite il y a les/a réduction des concepts en symboles <universellement compris>, et la manipulation identique de ces symboles en toute langue.

- la structure même du langage suppose l'expérience du vécu.

- Le savoir comme certitude visuelle

[Recherche d'expressions équivalentes à l'expression posée en enjeu dans l'article : tâtonnements et paradigmes lexicaux par changements des déterminants qui accompagnent le terme retenu et stable]

\subsubsection{Rôle des déterminants}

Mais une fois entré dans la rédaction de B1, si le terme « expérience » s'impose il n'est pas pour autant conceptuellement stabilisé. Ainsi dans le f 508 suivant, le terme est répété trois fois, il est testé, ruminé pour trouver sa place propre, cependant, le propos reste bien confus, même sans rature. Le terme "expérience" reste stable mais il est testé à nouveau avec différents déterminants. 


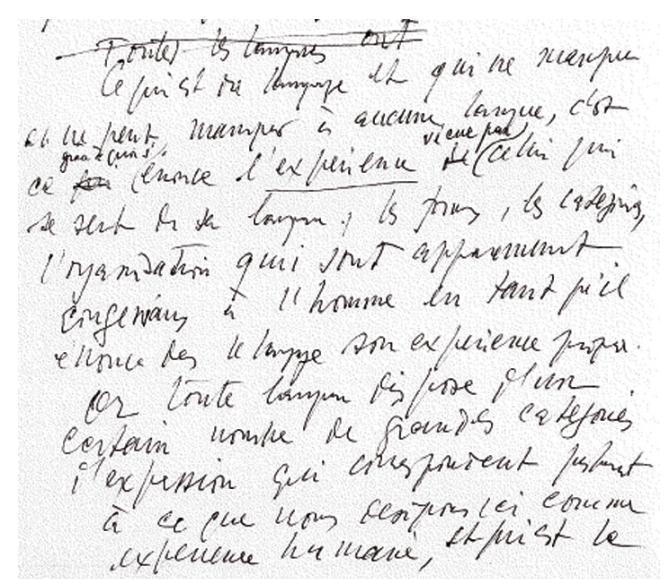

\begin{abstract}
Foutes les langues ont
Ce qui est du langage et ne manque et qui ne peut manquer à aucune langue, c'est ce qui <grâce à quoi s'>énonce l'expérience de <vécue par> celui qui se sert de la langue. , les formes, les catégories, l'organisation qui sont apparemment congéniaux à l'homme en tant qu'il énonce ds le langage son expérience propre.

Or toute langue dispose d'un certain nombre de grandes catégories d'expression qui correspondent justement à ce que nous désignons ici comme expérience humaine, et qui est le
\end{abstract}

\section{[Recherche d'expressions équivalentes par changements de déterminants]}

Voyons la suite immédiate au folio suivant 509 (p. 7 de B1)

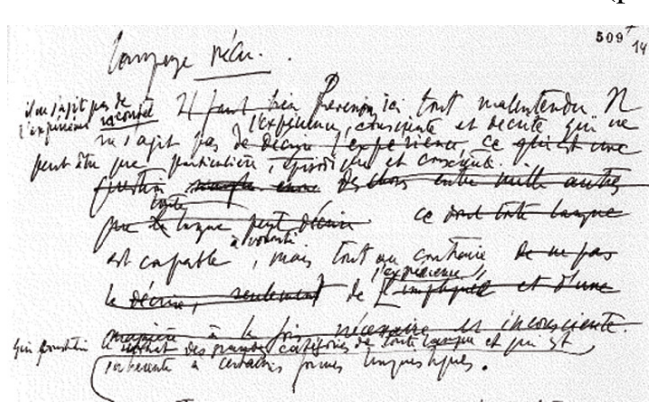

langage vécu.

Il faut bien <Il ne s'agit pas de l'expérience

racontée > Prévenons ici tout malentendu. Il ne s'agit pas de décrire l'expérience, <l'expérience, consciente et décrite qui ne peut être que particulière, épisodique consciente> te qui est une question simple encore des choses entre mille autres que la <toute> tangue peut décrire ce tont langue est capable $<\grave{a}$ volonté $>$ mais tout au contraire de ne pas la décrire, seulement de <l'expérience $\rangle$ impliquée et d'une manière à la fois nécessaire et inconsciente. $<q u i$ constitue le substrat des grandes catégories de toute langue et qui est $>$ inhérente à certaines formes linguistiques

La rédaction est laborieuse. Ici, ce sont les ratures et ajout successifs qui permettent à la conceptualisation de frayer sa voie. Mais des marques graphiques sont ajoutées, le soulignement est utilisé, comme une marque d'objet à cerner, à différentier : langage vécu/expérience racontée ; décrire

Notons ce remarquable passage où la recherche de... ce qu'il faut faire de la notion d'expérience ne trouve pas son mot: «Il ne s'agit pas de décrire l'expérience [...] mais tout au contraire de ne pas la décrire, seulement de » et qui finit par ne s'inscrire qu'en négatif : «ne pas », « ne pas-» à quoi s'ajoute la négation que constituent les ratures.

[Hésitations méthodologiques]

\subsubsection{Hésitations rédactionnelles et trouvailles}

Après tout ce mouvement de rature, ajout, reprise, Benveniste retombe sur ses pieds de théoricien et énonce une définition de l'expérience, définition constituée par le dernier ajout de ce fragment qu'il insère dans du déjà écrit conservé : «l'expérience <qui constitue le substrat des grandes catégories de toute langue> »

[Définir enfin]

Le rattrapage du fil énonciatif, de la linéarité discursive, par Benveniste, sur ce fragment est remarquable, mais il est assez courant de le trouver dans ses brouillons.

Tous les exemples précédents font partie de cet ensemble des sept folios qui ne seront pas repris dans B2 et seulement réduits en un paragraphe. Cependant, le passage de B1 à B2 reste encore riche du point de 
vue de cette notion. Les nombreux repentirs en B1 $\left(f^{\circ} 510\right)$ et le passage de B1 à B2 ( $\left.f^{\circ} 64\right)$ témoignent encore de la difficulté à énoncer théoriquement la notion d'expérience :

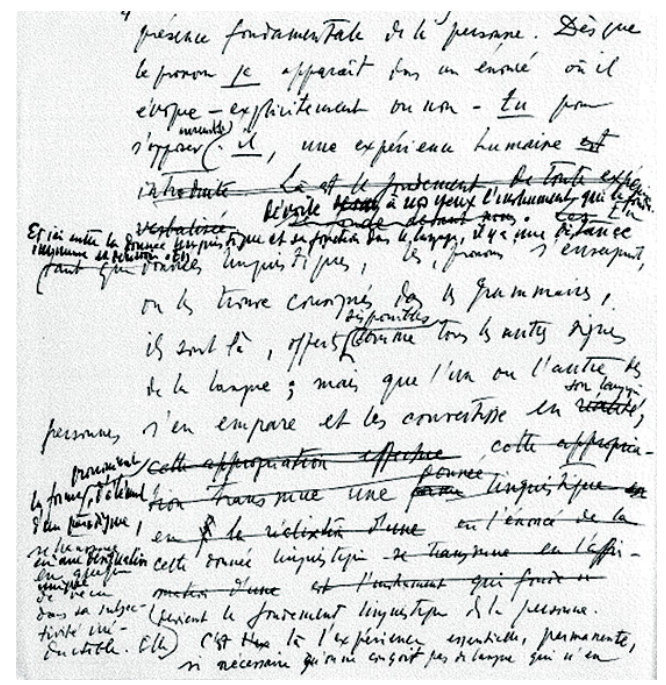

B1

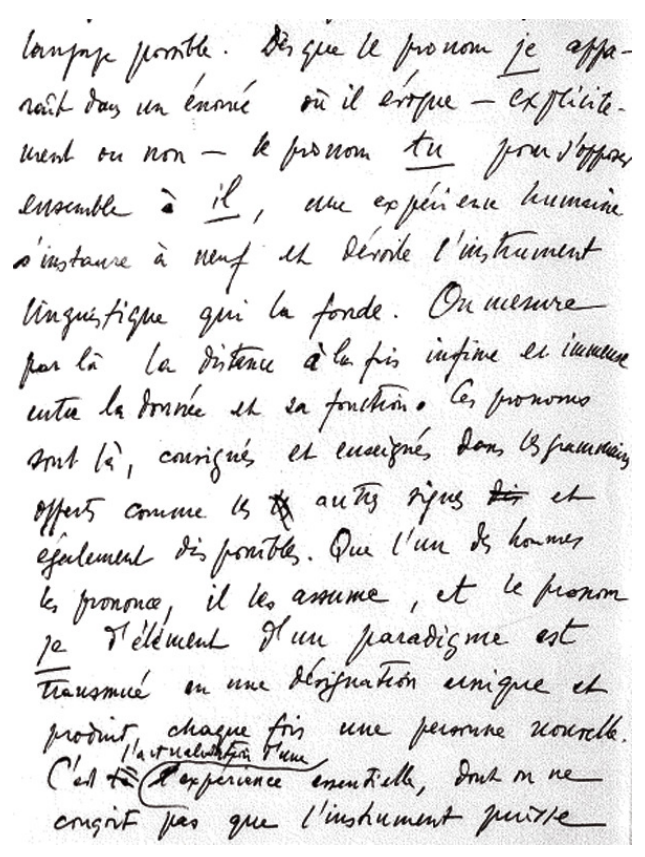

B2
[...] Dès que le pronom je apparaît dans un énoncé où il évoque explicitement ou non - $\underline{t u}$ pour s'opposer <ensemble> à il, une expérience humaine est introduite. Là est le fondement de toute expérience verbaliste se fonde devant nous < dévoile deva à nos yeux l'instrument qui la fonde>. Les <En <Et ici entre la donnée linguistique et sa fonction dans le langage, il y a une distance immense et dérisoire. En> tant que> données linguistiques, les pronoms s'enseignent, on les trouvent consignés dans les grammaires, ils sont là, offerts <disponibles $>$, comme tous les autres signes de la langue; mais que l'un ou l'autre <des personnes $>$ s'en empare et les convertisse en réalité <son langage > <la forme <pronominale> d'élément d'un paradigme se transmue en une désignation unique en quelque de vécu dans sa subjectivité
[...] Dès que le pronom je apparaît dans un énoncé où il évoque explicitement ou non - le pronom tu pour s'opposer ensemble à $\underline{i l}$, une expérience humaine s'instaure à neuf et dévoile l'instrument linguistique qui la fonde. On mesure par là la distance à la fois infime et immense entre la donnée et sa fonction. Ces pronoms sont là, consignés et enseignés dans les grammaires offerts comme les tes autres signes dis et également disponibles. Que l'un des 


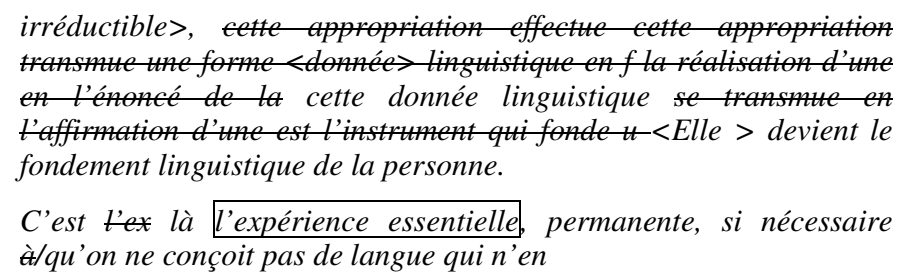

hommes les prononce, il les assume, et le pronom je d'élément d'un paradigme est transmué en une désignation unique et produit chaque fois une personne nouvelle. C'est łà $<$ l'actualisation d'une $>$ E'expérience essentielle, dont on ne conçoit pas que l'instrument puisse

Franck Neveu (2007b, 138) note «l'impossibilité de stabiliser les acceptions lorsque l'on a affaire à des termes issus de la langue commune » dans l'usage du métalangage linguistique. Nous sommes, ici, devant la même difficulté pour le choix d'un terme théorique : comment choisir un terme de la langue commune pour désigner, représenter une notion spécifique ayant poids de concept dans un discours théorique ? On voit que le terme, bien que choisi et stable, c'est à dire maintenu, donne lieu à des hésitations presque infinies quant à son extension et à sa compréhension.

Je voudrais enfin montrer combien ce qui se joue au cours de la scription pensante laisse des traces ... dans l'œuvre : justification, s'il en était besoin de ce typa d'études. En effet, dans B1 ( $\left.f^{\circ} 515\right)$ apparaît liée à la notion d'expérience l'expression « appareil formel » :

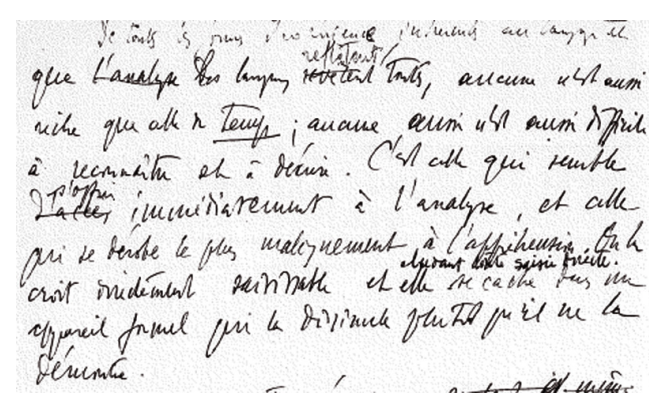

De toutes les formes d'expérience inhérentes au langage et que l'analyse d/les langues révèlent $<$ reflètent> toutes, aucune n'est aussi riche que celle du temps; aucune n'est aussi difficile à reconnaître et à décrire. C'est celle qui semble d'accès immédiatement à l'analyse, et celle qui se dérobe le plus malignement à l'appréhension. On la croit directement saisissable et <éludant toute saisie directe $>$ elle se cache dans un appareil formel qui la dissimule plutôt qu'il ne la démontre.

Mohammad Djafar Moïnfar n'a pas consulté les brouillons pour constituer l'édition des PLG 2, bien entendu, puisque les articles recollés avaient tous déjà été publiés. A-t-il eu la préscience ? Est-ce un pur hasard? Il se trouve que «L'appareil formel de l'énonciation », écrit cinq ans après «Le langage et l'expérience humaine », se trouve, dans le volume, directement à sa suite immédiate.

\section{Pour une éventuelle modélisation}

\subsection{Réserves et remarques générales}

Avec toutes les précautions nécessaires : un seul exemple, un seul auteur, un auteur fondateur, toutes caractéristiques ne permettant pas de tirer une loi générale, nous pouvons, néanmoins, tenter de rendre clair les résultats de cette étude sous une forme modélisable. Plus exactement, nous pouvons faire entrer les opérations de l'écriture scientifique linguistique relevées sur ce corpus particulier, dans un schéma modélisant.

Pour ce qui est de la genèse générale de l'article « le langage et l'expérience humaine », il semblerait que la recherche du titre de l'article, par Benveniste, est clairement liée à l'énonciation de l'objectif de l'article - ce qui, bien évidemment, n'a rien d'étonnant - mais qu'il fait cependant l'objet d'une lente rumination où se cherchent des déterminants spécifiques à partir de définitions basiques.

Pour ce qui est, plus particulièrement, du processus d'écriture et de mise en texte, l'auteur linguiste ne trouve pas immédiatement sa position énonciative, il la cherche et l'essaie : la linguistique devient le linguiste, puis il disparaît dans des propositions impersonnelles de type théorique, puis il réapparaît mais 
dédoublé, se distanciant, lui théoricien, du seul «linguiste descripteur », puis s'inclut dans un "nous » qui s'adresse à ses pairs et enfin redisparaît dans une proposition théorique impersonnelle sans "sujet" mais cette fois réappropriée et signée. Lorsque l'on observe ainsi de près la façon dont a été généré l'incipit de cet article, il est frappant de noter que Benveniste va passer d'une recherche épistémologique : le regard du linguiste, sa position, son point de vue, à une position de scripteur non marqué. Il ne déictique plus la posture du linguiste, il ne se désigne plus lui-même comme linguiste; il entre, en linguiste, dans le vif du sujet.

\subsection{Repères pour une modélisation}

Au delà de ces remarques générales et génétiques c'est à dire relevant d'un ordre chronologique d'écriture, je tenterai de "ranger" les opérations permettant la conceptualisation du terme "expérience" relevées au cours de cette étude. J'ouvre ainsi trois rubriques, dont les frontières, bien évidemment, ne sont pas étanches mais qui permettront d'encadrer les remarques dispersées au cours de l'analyse qui précède. Ces rubriques permettent aussi un premier classement des marqueurs linguistiques d'opérations.

\subsubsection{Processus scripturaux (plus ou moins contrôlés) et rédactionnels}

- Pointer le terme qui fait enjeu théorique (2.1.1) : on y trouve la marque du soulignement, dans notre cas, il s'agit de soulignement au sens propre, mais on sait que d'autres matérialités sont possibles : guillemets, italique...

- Resserrer la détermination des termes employés pour la construction théorique, premier pas vers une définition (2.1.1) : on trouve un lexique approprié à la détermination : «signifier », « définir » et l'usage de participes marquant l'accompli

- Recherche d'expressions équivalentes substitutives à l'expression posée en enjeu dans l'article: tâtonnements et paradigmes lexicaux par changements des déterminants qui accompagnent le terme retenu et stable (2.2.1): usages de paradigmes: «expérience vécue / propre / humaine / racontée / consciente et décrite / impliquée ». Cette étape est relevée par deux fois dans notre analyse (2.2.1 et 2.2.2), autrement dit elle est réitérée. Elle est marquée à la fois et en même temps par l'insistance et l'hésitation. Elle est le lieu de travail le plus intense, elle est le lieu de pensée le plus créatif.

- Définir enfin (2.2.3) : grâce à la locution «il ne s'agit pas de [...] mais tout au contraire » et de l'ajout d'une proposition déterminative du terme à définir «l'expérience qui constitue.. ; »

Il semblerait que l'ensemble de ces opérations se rapportent à des engagements terminologiques visant à satisfaire les exigences formelles déterminées par Swiggers $(1999,31)$ : transparence, adéquation, cohérence, économie. Nous sommes face, ici, à des essais de «calibrage » (Swiggers, 1999, 32) des différentes formes d'expression possibles qui permettront l'établissement d'une «terminographie » (Neveu, 2007b, 133) qui, dans le texte final de l'article restera implicite. L'analyse des brouillons la montre à l'œuvre, en train d'être agie, éprouvée par le linguiste.

\subsubsection{Procédés programmatiques (contrôlés)}

- Enoncer un programme d'opérations méthodologiques (2.1.1) : par l'utilisation d'infinitifs marquant l'injonction

- Différencier les tâches, les méthodes, les points de vue sur un même objet linguistique (2.1.2) par un lexique approprié : substantifs («tâche», «approche»), verbes («aborder», «s'attacher à », «prendre »), adverbes (« en tant que »)

- Se détacher de «l'habitude » (2.2.2) «manière habituelle », « négliger par habitude »

- Hésitations méthodologiques (2.2.2) : hésitation entre une tâche programmatique («il ne s'agit pas de décrire l'expérience $[. .$.$] mais au contraire de ne pas la décrire seulement... ») et la désignation de l'objet$ 
d'observation («il ne s'agit pas de l'expérience consciente [...] mais tout au contraire de l'expérience impliquée-»). L'usage de «il s'agit/il ne s'agit pas » s'impose là aussi.

- Enoncer fermement sa tâche (2.1.2): usage de la litote «nous essayons ici d'éclairer deux catégories... » où l'on remarque la présence incontournable de l'adverbe ici

Le linguiste laisse apparaître dans ses "brouillons" toutes les sollicitations méthodologiques par lesquelles il passe et les prescriptions, voire injonctions qui en résultent en vue de la finalisation de son discours. Nous remarquons que ce que nous avons appelé les "procédés programmatiques» se situent presqu'exclusivement en début de phase d'écriture, ce qui n'est guère étonnant; il est cependant intéressant de le noter car il s'agit là, peut-être d'un trait spécifique à l'entreprise scientifique.

\subsubsection{Positionnement épistémique de l'auteur}

- Marquer sa place particulière d'auteur à partir d'un héritage commun (2.1.1) : expression récurrente de «il s'agit de » et de l'adverbe de lieu «ici»

- Se situer comme auteur à l'intérieur d'une communauté pour affirmer ce qui veut apparaître comme un constat valable pour la communauté (2.1.2) : emploi de pronoms correspondant à la première personne $d u$ pluriel ; le nous/notre/nos inclusifs

- Marquer la tâche particulière de l'auteur (2.1.2), à l'intérieur de la communauté par emploi de tournures impersonnelles avec irruption d'un nous exclusif («nous essayons ici d'éclairer deux catégories...»)

- Marquer son option théorique singulière à l'intérieur d'une communauté (2.1.2)

\section{Conclusion}

Les travaux de genèse portant sur des corpus de manuscrits de linguistes sont encore rares. J'excepte, bien évidemment, les nombreux travaux concernant les manuscrits de Saussure mais force est de constater que ceux-ci ont pour la plupart (pas tous) pour finalité l'édition d'un texte. Je voudrais, ici, comme je l'ai dit, mettre les résultats de l'observation des "brouillons" au service de la compréhension de l'écriture scientifique et en particulier linguistique. Cela ne va pas de soi et nécessite de faire un effort : ne plus lire linéairement, en continu, un texte fini, publié, signé, assumé, mais accepter de tituber entre des linéarités superposées; se positionner dans l'espace d'un temps d'écriture et non pas dans un temps de lecture. En effet, lire un texte clos, c'est s'engager dans une durée de lecture, qui appartient au lecteur, qui est plus ou moins continue et qui permet de suivre une argumentation qui sera jugée sur la cohérence de son avancée, directement manifestée par le discours qui énonce. Exhumer des traits constitutifs de l'écriture processuelle d'un discours théorique signifie accepter de ne pas aller directement au discours fini et linéaire; ne pas lire mais suivre, à la trace, les hésitations, résistances, insistances d'une textualisation en train de se faire sous nos yeux.

Recueillir les traces manuscrites et les observer a pour principal mérite de compliquer la tache du linguiste pour ce qui est de la description énonciative mais aussi, et en même temps, de faire apparaître les processus mêlés, intriqués, de scription et de pensée ou... de pensée et de scription, d'énonciation ainsi que son effet direct sur l'énoncé.

De la même façon que le rêve n'est accessible que par le récit du rêve (rêve et pensée sont pareillement d'une matérialité spécifique, instable, insaisissable), la saisie de la pensée théorisante ne peut s'espérer que par la mise en mots et par leur énonciation en énoncé. Dans le domaine de l'écrit, c'est l'écriture en train de se faire qui inscrit la pensée qui ne préexiste pas à cette inscription, plus exactement qui l'articule afin de pouvoir être lisible et transmissible. Car qu'est-ce qu'écrire un «article» (du latin articulus : 
articulation) scientifique sinon articuler des concepts pour faire avancer une réflexion et articuler cette réflexion (cet article) à d'autres. Voici ce que l'on trouve sur le $f^{\circ} 504$ de B1 :

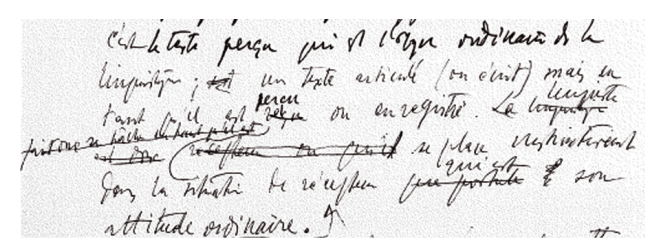

C'est le texte perçu qui est l'objet ordinaire de la linguistique; c'est un texte articulé (ou écrit) mais en tant qu'il est reçu <perçu> ou enregistré. La linguistique <le linguiste> est done <fait done sa tâche en tant qu'il est> récepteur ou qu'il se place instinctivement dans la situation de récepteur que postule <qui est> son attitude ordinaire.

Benveniste remplacera, par la suite, le terme «texte» par «discours» plus approprié et dont on sait aujourd'hui qu'il aura été le socle de la linguistique générale du théoricien.

Ainsi naissent les notions théoriques, ainsi c'est l'écriture elle-même qui est pensante, par sa rumination ou par sa fulguration, par la rature ou par la répétition, par tous les repentirs divers et multiples qui tissent la textualisation et qui habitent, qui continuent d'habiter le texte théorique final qui sera transmis pour être à nouveau articulé à d'autres pensées et réflexions.

L'observatoire avant-textuel de l'écriture linguistique en train de se fabriquer complète utilement «l'observatoire discursif » (Neveu, 2007b, 145) sur les textes de sciences du langage une fois produits. En effet, l'observation de l'écriture en production, en train de se nouer sur une suite de notes et brouillons, ne peut qu'être un facteur de développement des résultats obtenus par l'analyse des textes finis.

Je notais en introduction l'importance du fonds Benveniste pour l'histoire de la linguistique et pour la compréhension linguistique elle-même puisqu'elle met au jour la fabrication conceptuelle. Il faut ici noter combien l'observation de corpus scientifique est pertinente pour la compréhension du processus d'écriture en général et de l'écrit, écriture, écrit qui eux-mêmes sont des objets d'investigation linguistique.

\section{Références bibliographiques}

Adam J.-M. (2002). Discours et interdisciplinarité. Benveniste lecteur de Saussure, Cahiers Ferdinand de Saussure, 54, Genève : Droz, 241-258.

Bellemin-Noël, J. (1972). Le texte et l'avant-texte. Paris : Larousse.

Benveniste E. (1966 et 1974). Problèmes de linguistique générale, vol. 1 et 2, Paris : Gallimard.

Bergounioux G. (2005). Saussure sans sémantique ou le signifié ne fait pas science. Revue de sémantique et pragmatique 18, Presses Universitaires d'Orléans, 69-85.

Bergounioux G. (2007). La stylistique: Bally auteur du Cours de linguistique générale. Pratiques, 135-136, Questions de style, Metz.

Blumenthal P. (2007). Sciences de l'Homme vs sciences exactes: combinatoire des mots dans la vulgarisation scientifique. Revue française de linguistique appliquée, XII-2, Lexique et écrits scientifiques, Amsterdam : De Werelt, 15-28.

Boch F., Grossmann F. et Rinck F. (2007). Conformément à nos attentes...: les marqueurs de convergence/divergence dans l'article de linguistique. Revue française de linguistique appliquée, XII-2, Lexique et écrits scientifiques, Amsterdam : De Werelt, 109-122.

Brunet E. (2007). Le fonds Benveniste de la Bibliothèque nationale de France : rapport d'étape, BnF (synthèse en ligne : http://www.item.ens.fr/index.php?id=200861).

Fenoglio I. (dir). (2007). L'écriture et le souci de la langue. Ecrivains, linguistes : témoignages et traces manuscrites, Louvain-la-neuve : Academia-Bruylant. 
Fenoglio I. (2007). Du texte avant le texte. Formes génétiques et marques énonciatives de pré-visions textualisantes. Langue française, 155, 8-34.

Fenoglio I. (2008). Observer un manuscrit. Transmettre un document de genèse, in Crasson A. (ed) L'édition du manuscrit. Du manuscrit de création au scriptorium électronique, 53-64.

Fenoglio I. (2009a). Les notes de travail d'Emile Benveniste: où la pensée théorique naît via son énonciation. Langage \& Société 127 : Ecritures scientifiques. Carnets, notes, ébauches, Paris : MSH, 23-49.

Fenoglio I. (2009b). Conceptualisation et textualisation dans le manuscrit de l'article «le langage et l'expérience humaine » d'Emile Benveniste. Une contribution à la génétique de l'écriture en sciences humaines. Modèles linguistiques, Tome XXX-1, vol. 59, 71-99.

Fenoglio I. (2010) "Déplier l'écriture pensante pour relire l'article publié. Les manuscrits de «L'appareil formel de l'énonciation »" in Relire Benveniste. Des linguistiques énonciatives, (E. Brunet et R. Mahrer ed), Louvain la Neuve, Academia Bruylant (coll. «Sciences du langages »), à paraître.

Fonds Emile Benveniste de la BnF, Département des manuscrits (archives et manuscrits)

Genesis 20 (2003). Ecriture scientifique, Paris, éd. J.-M. Place.

Grossmann F. et Rinck F. (2004). La surénonciation comme norme du genre : l'exemple de l'article de recherche et du dictionnaire en linguistique. Langages 156, p. 34-50.

Langages 147 (2002). Processus d'écriture et traces linguistiques. Paris, éd. Larousse.

Langage et Société 103 (2003), Écritures en acte et genèse du texte.

Langue française 155 (2007), Avant le texte : les traces de l'élaboration textuelle. Paris, éd. Larousse.

Langage et Société 127 (2009) Ecritures scientifiques. Carnets, notes, ébauches.

Lazard G. (1999). Pour une terminologie rigoureuse : quelques principes et propositions, In Siouffi G. et Steuckardt A. Les linguistes et la norme. Aspects normatifs du discours linguistique, Peter Lang, 111-132.

Lebrave J.L. (2004). Pourquoi la critique génétique? Le point de vue d'un linguiste. In Matsuzawa K. Le texte et ses genèses. 21th Century COE Program International Conference Series $N^{\circ} 3$. Textes réunis et présentés par Graduate School of Letters, Nagoya University, 43-48.

Modèles linguistiques (2009). Tome XXX-1, vol. 59, Génétique de la production écrite et linguistique, La Garde.

Neveu F. (2007a). Singularités langagières du discours scientifique : l'exemple du discours linguistique. Pratiques 135-136, Questions de style, 101-118.

Neveu F. (2007b). Les fondements normatifs de la terminologie linguistique et l'observatoire discursif de la science du langage. In Siouffi G. et Steuckardt A. Les linguistes et la norme. Aspects normatifs du discours linguistique, Peter Lang, 123-148.

Ono A. (2007). La notion d'énonciation chez Emile Benveniste, Limoges : Lambert-Lucas.

Pratiques, 135-136 (2007). Questions de style, Metz : CRESEF.

Pratiques, 143-144 (2009). Ecrits de savoir, Metz : CRESEF.

Rey-Debove J.(1978). Le métalangage, Le Robert.

Rinck F. (2005). Images of scientific activity through the research article : a comparison between linguistics and literary studies in Flottum K. and Korsnes O., Academic Prosa 3, University of Bergen, 76-86.

Rinck F. (2006). Ecrire au nom de la science et de sa discipline. Les figures de l'auteur dans l'article en sciences humaines. Sciences de la société, 67, 94-111.

Swiggers P. (1983). Qu'est qu'une théorie (en) linguistique ? Modèles linguistiques, T 1, vol. 5, 3-15.

Swiggers P. (1999). Pour une systématique de la terminologie linguistique: considérations historiographiques, méthodologiques et épistémologiques, Mémoires de la société de Linguistique de Paris, Nouvelle série, Tome VI : La terminologie linguistique, Peeters, 11-49. 
Valette M. (2006). La genèse textuelle des concepts scientifiques. Etude sémantique sur l'œuvre du linguiste Gustave Guillaume. Cahiers de lexicologie, 89, 125-142.

${ }^{1}$ Le texte et l'avant-texte, 1972, 15.

2 "Reproduire le manuscrit, présenter les brouillons, établir un avant-texte" in Littérature 28, 1977, 8.

${ }^{3}$ Je désigne par "note" des notes de travail, autrement dit, toute formulation inscrite par Emile Benveniste et entrant, d'une manière ou d'une autre, dans le processus d'écriture de l'article. Il ne s'agit pas de prises de notes exogènes mais de notes endogènes, prises, ou reprises, dans le mouvement de travail que constitue l'écriture scientifique. Ces notes de travail se présentent sous toutes sortes de formes : note d'une référence, réflexion abrégée, fragment très rédigé et sur n'importe quel type de support: verso de lettre, de carton d'invitation, petites feuilles de carnet, grande feuille volante, etc. Elles se tiennent parfois sur un seul feuillet, parfois sur plusieurs qui se suivent.

${ }^{4}$ Il s'agit du fonds Meillet du Collège de France aujourd'hui confié et consultable à l'IMEC (Institut mémoires de l'Edition Contemporaine, Caen) comme l'ensemble des archives du Collège de France.

${ }^{5}$ Cote BnF : PAP OR 46, env.139, fo 496 à 503.

${ }^{6} \mathrm{Il}$ est possible que l'on en retrouve ailleurs, ses notes pouvaient servir à plusieurs projets d'écriture $(C f$. Fenoglio, 2009a et b).

${ }^{7}$ Cote BnF : PAP OR 46, env.139, $f^{\circ} 496$ et $f^{\circ} 504$ à 535.

${ }^{8}$ Cote BnF : PAP OR 50, env. 179, fo 47 à 95. 